\title{
Stabilization of Self-Coherent OFDM with Injection Locked Laser
}

\author{
Susmita Adhikari $^{(1)}$, Stylianos Sygletos ${ }^{(2)}$, Andrew D. Ellis ${ }^{(2)}$, Beril Inan $^{(3)}$, \\ Sander L. Jansen ${ }^{(4)}$ and Werner Rosenkranz ${ }^{(1)}$ \\ 1: Chair for Communications, Christian-Albrechts-Universität zu Kiel, Kiel, Germany (asu@tf.uni-kiel.de) \\ 2: Tyndall National Institute and Department of Physics, University College Cork, Ireland \\ 3: Department of Communications Engineering, Technical University of Munich, Munich, Germany \\ 4: Nokia Siemens Networks, Munich, Germany
}

\begin{abstract}
With the rebirth of coherent detection, various algorithms have come forth to alleviate phase noise, one of the main impairments for coherent receivers. These algorithms provide stable compensation, however they limit the DSP. With this key issue in mind, Fabry Perot filter based self coherent optical OFDM was analyzed which does not require phase noise compensation reducing the complexity in DSP at low OSNR. However, the performance of such a receiver is limited due to ASE noise at the carrier wavelength, especially since an optical amplifier is typically employed with the filter to ensure sufficient carrier power.

Subsequently, the use of an injection-locked laser (ILL) to retrieve the frequency and phase information from the extracted carrier without the use of an amplifier was recently proposed. In ILL based system, an optical carrier is sent along with the OFDM signal in the transmitter. At the receiver, the carrier is extracted from the OFDM signal using a Fabry-Perot tunable filter and an ILL is used to significantly amplify the carrier and reduce intensity and phase noise. In contrast to CO-OFDM, such a system supports low-cost broad linewidth lasers and benefits with lower complexity in the DSP as no carrier frequency estimation and correction along with phase noise compensation is required.
\end{abstract}

Keywords: OFDM, injection locking, self-coherent, phase noise, Fabry-Perot tunable filter.

\section{INTRODUCTION}

The demand of ubiquitous high-speed access to information has led to extensive research in advanced modulation formats. Among others, orthogonal frequency division multiplexing (OFDM) has received considerable interest because of the possibility to scale to higher level modulation formats and to realize next generation agile optical networks [1]. With the rekindled interest in coherent receivers, much of the effort has been focused on the digital signal processing (DSP) which is a key technology for realizing $400 \mathrm{~Gb} / \mathrm{s}$ and beyond optical transmission systems. Recently, a transmission of $40 \times 117.6 \mathrm{~GB} / \mathrm{s}$ conventional coherent optical OFDM (CO-OFDM) over 10,181 km hybrid large core and ultra-low loss fibers has been demonstrated that employs coherent detection and DSP [2]. Though coherent detection offers a reception sensitivity that is higher than the direct detection (DD) receiver, it is inherently prone to laser phase noise, especially when scaling to higher quadratic-amplitude modulation (QAM) constellations. In [3], the authors use RF-pilot along with pilot subcarriers to mitigate the effect of phase noise. The RF-pilot based phase noise compensation (RF-PNC) was first introduced in [4] where phase-noise compensation is realized by placing a RF-pilot (RFP) tone in the center of the OFDM transmit spectrum, which is subsequently used at the receiver in order to undo phase noise impairments. Nevertheless, the complexity of implementing RF-PNC increases the requirements of the DSP, which is already limited in speed and processing capabilities.

An interesting alternative is self coherent optical (SCO-) OFDM [5]. SCO-OFDM is similar to the RF-PNC concept for CCO-OFDM, except that the pilot is extracted in optical domain and used as local oscillator (LO) using heterodyne or homodyne detection to reverse the phase noise effect. No phase noise compensation is required in the DSP, consequently reducing the DSP complexity. No LO is required at the receiver, reducing the cost of an extra LO. However, the performance of such a receiver is limited due to ASE noise at the carrier wavelength, especially since an optical amplifier is required at the receiver to ensure sufficient power for selfcoherent detection [6]. In [7], the use of an injection-locked laser (ILL) to retrieve the frequency and phase information from the extracted carrier without the use of an amplifier was proposed. ILL based self coherent OFDM (ILL-OFDM) detection is realized by extracting the carrier from the OFDM signal using a Fabry-Perot tunable filter (FP-TF) and an ILL is used to significantly amplify the carrier and reduce intensity and phase noise. Unlike in [5] an amplifier is not required; the pilot carrier is enhanced using an ILL. In contrast to COOFDM, such a system supports low-cost broad linewidth lasers and benefits with lower complexity in the DSP as no carrier frequency estimation and phase noise compensation is required.

In this paper, a short introduction to injection locking is given followed by the illustration of the implementation of ILL-OFDM system and some experimental results that present the back-to-back and $400 \mathrm{~km}$ transmission performance. A negligible penalty of $0.5 \mathrm{~dB}$ is seen when compared to CO-OFDM and a significant improvement of $2.2 \mathrm{~dB}$ is observed when compared to SCO-OFDM. The nonlinear tolerance of such a system is similar to RFP implemented CO-OFDM. 


\section{INJECTION LOCKED LASER BASED CARRIER ENHANCEMENT}

Optical injection locking (OIL) has been proposed as a method to achieve laser synchronization many years ago. Since then, it has found a wide range of applications in different areas of photonics technology, i.e. for coherent detection in optical communication systems, ultra-soft pulse generation and microwave signal generation. Its operating principle is based on the fundamental laws of laser physics and oscillator dynamics. When a monochromatic signal is injected into a resonant circuit or self-sustained oscillator, at a frequency within a narrow range around its free-running frequency, then the injected signal can capture its oscillation behaviour, so that, the oscillator or circuit can be more or less controlled [8]. In optics this is usually understood by a masterslave laser pair. The master laser injects light into the slave laser and provided the difference between the free running frequencies of the two lasers is within certain limits, called the locking range, the slave laser will assume the same frequency as the master laser and become phase locked to it i.e. tracking the quantum phase fluctuations of the master and acquiring the same linewidth. The locking range of the optical injection locking mechanism can be in the order of $\mathrm{GHz}$, much larger than the $\sim \mathrm{MHz}$ linewidth of typical distributed feedback (DFB) lasers, which enables low phase error performance and robust operation at high symbol rates. This is a noticeable implementation advantage of OIL against alternative synchronization schemes such as optical phase lock loops (OPLLs), which has made them particular attractive for a number of recent demonstrations in phase sensitive amplification [9] and coherent detection [10]. Indeed, traditional OPLL circuits made from commercial off-the-shelf (COTS) fiber pigtailed components have large feedback delays limiting the obtainable loop bandwidth and setting restrictions on the summed laser linewidth of the transmitter (master) and the local oscillator (slave). Unless compact OPLLs are developed i.e. based on micro-optics or photonic integrated circuits, to enable loop delays of less than 100psec, expensive laser sources with a linewidth of a few $\mathrm{kHz}$ will have to be used in alternative to minimize BER degradations due to untracked phase errors [11]. Optical phase locking enabled carrier recovery is an inherently regenerative process. As a low power carrier or pilot tone is needed just for the phase synchronization of a high power laser, any amplitude distortions will be suppressed whilst only low bandwidth phase fluctuations allowed by the feedback process $(<1 \mathrm{GHz})$ will be transferred to the new carrier (LO). This has important practical implication in coherent detection process. The pilot tone can be selected very effectively from a modulated signal spectrum without affecting the quality of the local oscillator and any OSNR degradation will not be transferred to the recovered carrier. However, when optical injection locking considered its optimum operating conditions need to be identified to achieve the aforementioned regenerative characteristics for the optical carrier. Theoretical and experimental studies have already indicated that at low input powers the injection locking mechanism becomes highly selective and it provides large suppression of the amplitude fluctuations [12]. On the other hand, its locking range narrows down significantly and the mechanism becomes susceptible to environmental drifts. The spectral shape of the signal format carrying pilot tone clearly plays an important role here, and it is a subject of further study to identify the associated performance implication in coherent detection.

\section{EXPERIMENTAL SETUP FOR INJECTION LOCKED LASER AIDED SCO-OFDM}

The experimental configuration for the transmitter, link and receiver of the ILL-OFDM system is illustrated in Fig. 1. In the experiments, an arbitrary waveform generator (AWG) was used at a sampling rate of $10 \mathrm{GSamples/s}$ to generate a continuous baseband signal. The OFDM baseband waveform was calculated offline and uploaded into the AWG. A low-pass filter (LPF) after the AWG with $4.4 \mathrm{GHz}$ bandwidth was used to suppress any aliasing products. For the generation of the OFDM signal, a 256 FFT size was used, from which 148 subcarriers were used as data. The modulation format was a non-rectangular 8-QAM constellation on each

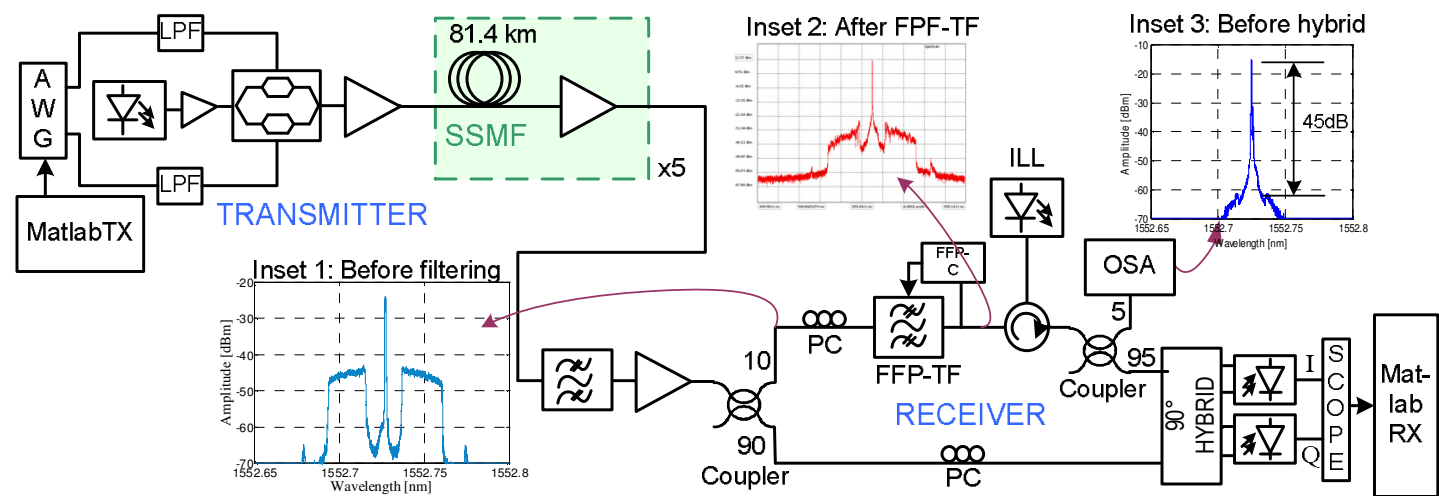

Fig. 1: Schematic of injection locked laser based self coherent optical OFDM system with Inset 1: optical spectrum before carrier extraction, Inset 2: optical spectrum after FPF-TF and Inset 3: optical spectrum after carrier extraction; FP-TF/C: Fabry-Perot tunable filter/controller, PC: polarization controller, ILL: injection locked laser, OSA: optical spectrum analyzer. 
subcarrier. 64 subcarriers around the DC were padded with zeros to make place for the insertion of an optical carrier/ pilot-tone. A cyclic prefix overhead of $6.25 \%$ was used to increase ISI tolerance. The net and nominal data rates were $\sim 14.9 \mathrm{~Gb} / \mathrm{s}$ and $\sim 15.3 \mathrm{~Gb} / \mathrm{s}$, respectively. A laser with specified linewidth of $100 \mathrm{kHz}$ was used at the transmitter to generate a continuous wave signal that was subsequently modulated with the OFDM signal by an IQ-Mach-Zehnder modulator (IQ-MZM). The IQ-MZM was biased such that the optical carrier was not totally suppressed at the transmitter leaving a carrier at the center of the spectrum as shown in the Inset 1 of Fig.1.
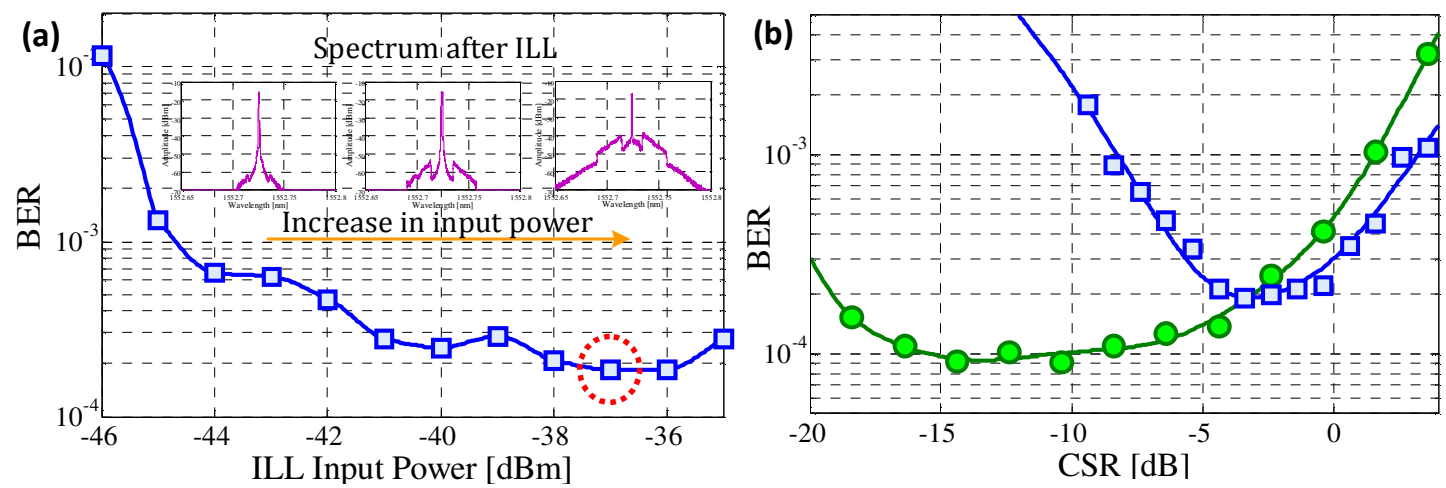

Fig. 2: BER curves at a required OSNR of $12 \mathrm{~dB}$ (a) as a function of ILL input power with CSR $=-4 \mathrm{~dB}$, (b) as a function of CSR for $\square$ ILL-OFDM with ILL Input Power $=-37 \mathrm{dBm}$ and $\bigcirc$ CO-OFDM
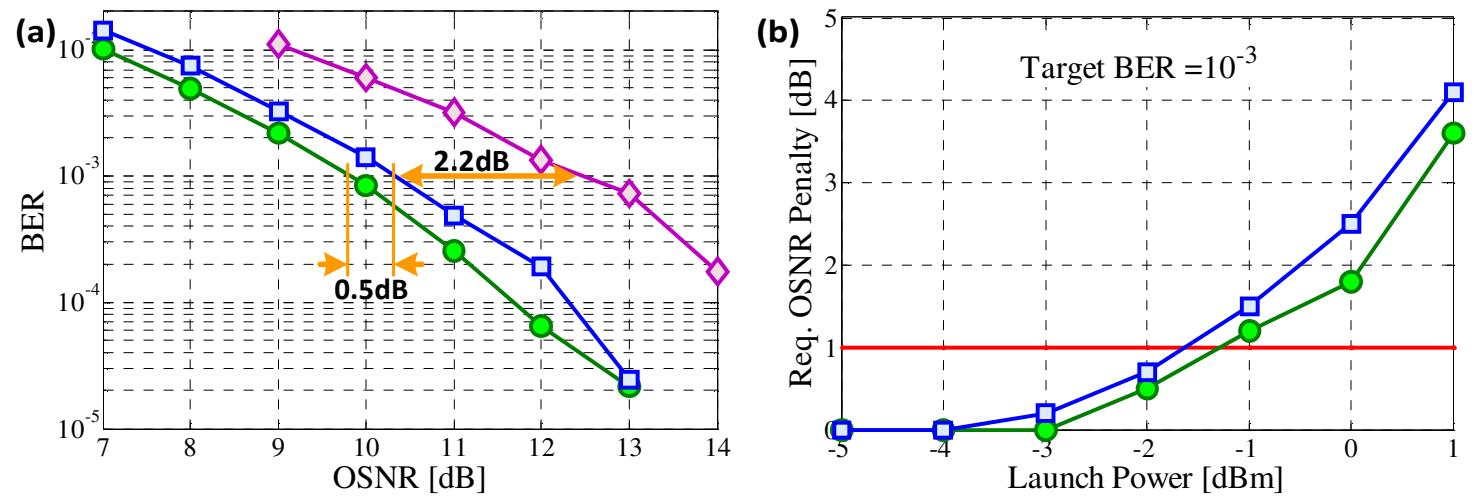

Fig.3: (a) BER as a function of OSNR for back-to-back scenario for $\square$ ILL-OFDM at ILL Input Power $=-37 \mathrm{dBm}$, $\mathrm{CSR}=-4 \mathrm{~dB}, 0$ CO-OFDM at CSR $=-10.2 \mathrm{~dB}$ and $\diamond$ SCO-OFDM at $\mathrm{CSR}=-5.4 \mathrm{~dB}$

(b) Required OSNR penalty as a function of the launch power after $400 \mathrm{~km}$ transmission for $\square$ ILL-OFDM at $\mathrm{CSR}=-4 \mathrm{~dB}$, ILL Input Power $=-37 \mathrm{dBm}$ and $O$ CO-OFDM at CSR $=-10.2 \mathrm{~dB}$

The transmission link consisted of 5 spans of 81.4-km standard single mode fiber (SSMF) without any dispersion compensation. After every span, an erbium-doped fiber amplifier (EDFA) was used for amplification. At the receiver, a coupler was implemented to separate the OFDM signal path from carrier extraction stage. In the carrier extraction path, a FP-TF filter with a 3-dB bandwidth of $120 \mathrm{MHz}$ was employed to separate the carrier from the OFDM signal. With this filter, a guardband of $1.3 \mathrm{GHz}$ was used in the experiment to easily filter out the optical carrier at the receiver. Note that the center wavelength of the FP-TF was aligned to that of the laser. In order to cope with laser drifts, an automated feedback loop was realized to actively tune the center frequency of the FP-TF to the optical carrier using an external Fabry-Perot controller. The spectrum after FP-TF is shown in Inset 2 of Fig. 1. Compared to the setup in [5], where a second FP-TF was used to suppress the OFDM signal leaking into the filtered carrier and amplifiers were used to amplify the carrier, in this setup only a single FP-TF was required. This is because an ILL was used to transfer the frequency and the phase information of the carrier to the laser via injection locking and enhance the pilot power. The ILL was an AlInGaAs multiple quantum well discrete-mode laser diode with rigid geometry [13]. In Inset 3 of Fig. 1, the optical spectrum after ILL is shown. It can be seen that after the ILL, the OFDM signal leaking into the filtered carrier was suppressed to more than $40 \mathrm{~dB}$ (measured at a resolution of $0.8 \mathrm{pm}$ ). The output of the ILL was then sent to the $90^{\circ}$ optical hybrid and used as LO. The polarization of the extracted optical carrier and optical OFDM signal were aligned with polarization controller in the signal path before detection. The bandwidth of the oscilloscope was $16 \mathrm{GHz}$ and the sampling frequency was $50 \mathrm{GSamples/s.} \mathrm{For} \mathrm{the} \mathrm{CO-OFDM} \mathrm{systems,} \mathrm{an} \mathrm{external} \mathrm{cavity} \mathrm{laser} \mathrm{(ECL)}$ with 100-kHz typical linewidth was used as LO and in the DSP, RF-PNC [1] was used. 


\section{EXPERIMENTAL RESULTS AND DISCUSSION}

In this section, the performance of ILL-OFDM is compared to that of CO-OFDM with RF-PNC scheme and SCO-OFDM. The power into the injection locked laser is a crucial parameter to be optimized for ILL-OFDM system. Fig. 2(a) shows the influence of input signal power to the laser on the bit-error-rate (BER) in a back-toback case with optical signal-to-noise ratio (OSNR) fixed at $12 \mathrm{~dB}$. For low input power two phenomena occurs. Firstly, since the laser does not have an isolator it is vulnerable to reflections. Secondly, the injection locking area also becomes extremely narrow such that, practically, the system is vulnerable to any frequency drifts that may originate from the transmit laser. Both these phenomena are responsible for increase in BER. The optimum value of $-37 \mathrm{dBm}$ is seen from the figure and is kept at this point for all the following results related to ILLOFDM. At powers above the optimum, due to leaking of OFDM signal into the laser, (as seen from the rightmost spectrum in the inset of Fig 2(a)) the BER increases. The power of the carrier with respect to the power of the OFDM signal, called carrier-to-signal ratio (CSR), has a strong influence on the system performance as well. In Fig. 2(b), the influence of CSR on the BER performance is shown in a back-to-back configuration. The OSNR in this measurement is kept constant at $12 \mathrm{~dB}$ and the optimum CSR is seen at $10.2 \mathrm{~dB}$ and $-4 \mathrm{~dB}$, for CO-OFDM and ILL-OFDM, respectively. When the CSR is below the optimum, the optical carrier is too weak and ASE noise limits the effectiveness of phase noise compensation. For high CSR, the relative power of the OFDM signal becomes too low and hence the performance gets worse. For all the following measurements, the CSR values are set to the optimum value.

Fig. 3(a) depicts the back-to-back BER performance as a function of the OSNR. The required OSNR for a BER of $10^{-3}$ is $9.8 \mathrm{~dB}$ and $10.3 \mathrm{~dB}$ for CO-OFDM and ILL-OFDM, respectively. Compared to SCO-OFDM [5], a 2.2-dB improvement is seen in ILL-OFDM. In SCO-OFDM, the extracted optical carrier is impaired by ASE noise arising from amplifiers. The increase in performance in ILL-OFDM can be attributed to the fact that ILL cleans up the carrier from the amplitude noise induced due to OSNR degradation and enhances its power without the need to amplify. Subsequently it can be said that ILL-OFDM does not only give a 2.2-dB benefit over SCOOFDM but also removes the requirement of a second FP-TF and amplifiers. Finally, Fig. 3(b) shows the nonlinear tolerance of CO-OFDM and ILL-OFDM after 400-km transmission. The launch power is varied from $5 \mathrm{dBm}$ to $1 \mathrm{dBm}$. The y-axis of the plot depicts the OSNR penalty for a BER of $10^{-3}$. As expected for low launch powers no OSNR penalty is present. As the launch power is increased, the nonlinearities come into play. Allowing a 1-dB penalty in required OSNR, the maximum tolerable launch power is found to be $-1.2 \mathrm{dBm}$ and $1.7 \mathrm{dBm}$ CO-OFDM and SCO-OFDM, respectively.

\section{CONCLUSIONS}

In this paper, the injection-locked laser based pilot enhancement scheme is discussed and compared to conventional coherent OFDM and self coherent OFDM. A residual penalty of $0.5 \mathrm{~dB}$ is seen when the performance of such a system is compared to conventional coherent OFDM and a drastic improvement of $2.2 \mathrm{~dB}$ is observed when compared to self coherent-OFDM. The system benefits from lower DSP complexity and does not require additional amplifiers.

\section{REFERENCES}

[1] S.L. Jansen, "Multi-Carrier Approaches for Next-Generation Transmission: Why, Where and How?", in Proc. OFC, OTh1B.1, 2012.

[2] S. Zhang, et al., "40x117.6 Gb/s PDM-16QAM OFDM Transmission over 10,181 km with Soft-Decision LDPC Coding and Nonlinearity Compensation", in Proc. OFC, PDP5C.4, 2012.

[3] D. Qian, et al., "101.7 Tb/s (370x294-Gb/s) PDM-128QAM-OFDM Transmission over 3x55-km SSMF using Pilotbased Phase Noise Mitigation", in Proc. OFC, PDPB5, 2011.

[4] S. L. Jansen, et al., "Coherent Optical 25.8-Gb/s OFDM Transmission Over 4160-km SSMF", J. Lightwave Technology, vol. 26, no. 1 pp. 6-15, Jan. 2008.

[5] S. Adhikari, et al., "Experimental Investigation of Self Coherent Optical OFDM Systems Using Fabry-Perot Filters for Carrier Extraction", in Proc ECOC, Tu.4.A.1, 2010.

[6] J. Arnaud, "Enhancement of optical receiver sensitivities by amplification of the carrier", IEEE JQE, vol.4, no.11, pp. 893- 899, 1968.

[7] S. Adhikari, et al., "Enhanced Self-Coherent OFDM by the Use of Injection Locked Laser", in Proc. OFC, 2012.

[8] A.E. Siegman, "Lasers", University Science Books, 1986.

[9] S. Sygletos, et al., "Phase synchronization scheme for a practical phase sensitive amplifier of ASK-NRZ signals", Optics Express, Vol. 19, Issue 13, pp. 12384-12391, 2011.

[10] S. K. Ibrahim, et al., "Novel real-time homodyne coherent receiver using a feed-forward carrier extraction scheme", Optics Express, Vol.19, Issue 9, pp. 8320-8326, 2011.

[11] A.C. Bordonalli, et al., "High-performance phase locking of wide linewidth semiconductor lasers by combined use of optical injection locking and optical phase-lock loop", J. Lightwave Technology, vol. 17 no. 2, Feb. 1999.

[12] E. K. Lau, et al., "Bandwidth Enhancement by Master Modulation of Optical Injection-Locked Lasers", J. Lightwave Technology, vol. 26, , no.15, 2584-2593, 2008

[13] B. Kelly, et al., "Discrete mode laser diodes with very narrow linewidth emission", Electronics Letters, Vol.43, Issue.23, 2007. 